\title{
Preliminary optical design for a 2.2 degree diameter prime focus corrector for the Blanco 4 meter telescope.
}

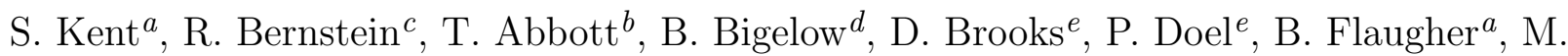 \\ Gladders $^{f}$, A. Walker ${ }^{b}$, and S. Worswick ${ }^{g}$ \\ ${ }^{a}$ Fermilab*, P. O. Box 500, Batavia, IL 60510; \\ ${ }^{b}$ CTIO/AURA ${ }^{\dagger}$, Casilla 603, La Serena, Chile S.A.; \\ ${ }^{c}$ Astronomy Dept., U. of Michigan, Ann Arbor, MI, 48109-1042; \\ ${ }^{d}$ Dept. of Physics, U. of Michigan, Ann Arbor, MI 48109; \\ e Optical Science Laboratory, Dept. Physics and Astronomy, University College London, \\ Gower Street, London WC1E 6BT, UK; \\ ${ }^{f}$ Carnegie Observatories, 813 Santa Barbara St, Pasadena, CA 91101; \\ g 1 Betony, Vale, Royston, Hertfordshire SG8 9TS, UK
}

\begin{abstract}
We describe a five element corrector for the prime focus of the 4 meter Blanco telescope at the Cerro Tololo Inter-American Observatory (CTIO) in Chile that will be used in conjunction with a new mosaic CCD camera as part of the proposed Dark Energy Survey (DES). The corrector is designed to provide a flat focal plane and good images in the SDSS $g, r, i$, and $z$ filters. We describe the performance in conjunction with the scientific requirements of the DES, particularly with regard to ghosting and weak-lensing point spread function (PSF) calibration.
\end{abstract}

Keywords: Dark Energy, Blanco, CTIO, Telescopes, Optics, Corrector

\section{INTRODUCTION}

The Dark Energy Survey (DES) is a proposed project to build a wide-field CCD camera for the prime focus of the 4 meter Blanco telescope at the Cerro Tololo Inter-American Observatory (CTIO) in Chile for the purpose of conducting an imaging survey of 5000 square degrees of sky. ${ }^{1}$ The survey will detect and measure over 300 million galaxies in four bandpasses out to redshift 1.3. The principal science goal is to study the nature of dark energy in the universe using four complementary techniques, including galaxy clusters, weak lensing, supernovae, and correlation functions. The survey will take 525 nights of observing spread out over five years. During the remainder of the time the instrument will be available to the general U.S. National Optical Astronomy Observatory (NOAO) user community The DES collaboration currently includes 14 institutions on 3 continents.

The survey instrument (called DECam) ${ }^{2}$ will consist of a CCD camera, widefield optics, and a mechanical structure that will replace the existing prime focus cage on the telescope. The new cage will retain the ability to flip and position a secondary mirror in the beam for the Cassegrain focal position. The camera will have a field of view about 8 times larger than the existing Mosaic II camera. The CCDs will be optimized for red sensitivity.

\section{REQUIREMENTS}

The principal requirements on the corrector are given in Table I. The top level requirements that drive these are a combination of science, operational, and technical considerations. The science program was designed assuming that the instrument performance would be comparable to the existing Mosaic II imager and telescope, aside from changes to the field of view and the CCD characteristics. The operational requirements are driven by the need to complete the program in 5 years with along with considerations of the desired limiting magnitude in each band.

* Operated by Universities Research Association Inc. under Contract No. DE-AC02-76CH03000 with the United States Department of Energy.

$\dagger$ Operated by Associated Universities for Research in Astronomy (AURA) Inc., under cooperative agreement with the National Science Foundation 
The observing plan includes a $25 \%$ continency to accommodate an event such as an El Nino weather pattern for one year. This requirement, in particular, drives the specification of a 2.2 degree diameter for the field of view.

A design philosophy within the project has been to rely on existing technology where possible, to minimize schedule risk and cost. For the optics, this philosophy guides us to limit ourselves to optical elements and materials for which similar parts can be found in other existing astronomical instruments.

The DES itself does not require ultraviolet (UV) imaging capability; however, the user community does have an interest in such capability, so the performance of the corrector at 0.35 microns is a consideration in the design.

A significant design decision is to not incorporate an atmospheric disperson compensator (ADC) in the corrector. An ADC adds cost and schedule risk to the project, complicates the optical design, and decreases throughput. The bulk of the DES observing time will be spent imaging in red and near-IR bands, where an $\mathrm{ADC}$ would be of little benefit. Additionally, for the weak lensing portion of the observing program, an ADC, even if available, would be a detriment in that it would complicate the interpretation of point spread function (PSF) patterns. The current ADC available for the Mosaic II camera is usually turned off by users. Finally, at least one other Southern Hemisphere wide field imager, that on VST, is optimized for blue performance with an $\mathrm{ADC}$, so this capability is at least present within the astronomical community.

Table 1. Principal DES Corrector Requirements

\begin{tabular}{lcl}
\hline Parameter & Value & Comment \\
Image Size & 0.27 arcsec & FWHM $r, i, z$ bands \\
Bandpasses & $g: 0.40-0.55 \mu$ & Refocus allowed for each bandpass \\
& $r: 0.56-0.71 \mu$ & \\
& $i: 0.70-0.85 \mu$ & \\
& $z: 0.83-1.00 \mu$ & \\
Pixel size & $15 \mu$ & Fixed by CCD design \\
Pixel scale & 0.27 to 0.30 arcsec & Natural scale at prime focus \\
Field diameter & $2.2^{\circ}$ & Cover survey area in 5 years \\
Focal plane & Flat & \\
Operating temperature & -5 to $+25 \mathrm{C}$ & CTIO conditions \\
Radiation from Glass & 0.01 event $\mathrm{cm}^{-2} \mathrm{sec}^{-2}$ & $\begin{array}{l}\text { Approximate cosmic ray } \\
\text { rate at sea level }\end{array}$ \\
Throughput & $75 \%$ & Includes primary mirror, \\
& reflection and transmision losses. \\
Pupil Ghost Gradient & $0.05 \% \mathrm{~mm}^{-2}$ & $3 \%$ gradient over length of one CCD \\
Halo Ghost & $25 \mathrm{mag} \mathrm{arcsec}^{-2}$ & $\begin{array}{l}\text { Maximum ghost surface brightness } \\
\text { from a } 6 \text { th mag star }\end{array}$ \\
& & \\
& &
\end{tabular}

The use of relatively thick CCDs means that we are considerably more sensitive to ionizing radiation sources, such as cosmic rays, than standard thinned CCDs. ${ }^{3}$ Cosmic ray muons constitute an irreducible background, so our requirement is that any additional source from the corrector should be no more than this flux at sea level. (At the elevation of CTIO, the cosmic ray flux itself is approximately doubled.) Many types of optical glass contain trace amounts of radioactive isotopes, such as K-40 or Th-232, either by design or as trace contaminants. When such glass is used as a window of a vacuum dewar, beta particles emitted from the window surface on the vacuum side that can strike a CCD and deposit charge. In particular, BK7, which contains about $10 \%$ potassium, makes it a poor choice for a window material. 


\section{DESIGN}

Several designs for correctors at the prime focus of 4 meter class telescopes with fields of order 2 degrees have appeared in the last several years. ${ }^{4,5}$ Additionally, two concept design studies were solicited by NOAO in conjunction with the solicitation of new instrumentation for the Blanco. ${ }^{6,7}$ These designs explore a variety of configurations and push the size limits of existing optics elements (both spheres and aspheres). We find, in common with others, that the simplest configurations with good image quality employ at least five powered elements and one or more aspheric surfaces. We are still iterating on a final design, but the preliminary design presented here embodies all of the major features that the final design will incorporate.

Prime focus correctors with little power tend to be self-achromatizing, so designs with a single glass type are feasible. If one could optimize the conic constant of the primary mirror, a shape close to parabolic would be preferred, ${ }^{5}$ but that is not an option here. In general, the image quality and field size both improve with the addition of lenses or aspheric surfaces and with an increase in the length (and thus element sizes) of the corrector; however they also increase the cost and construction time, and thus any corrector design represents a compromise among all these parameters and factors. We have explored much of this parameter space and have additional explored designs with mixed glass types.

A particular design is shown in Fig. 1. This design has five lenses (including the dewar window) and favored a smallish first element (C1) for the corrector (930 $\mathrm{mm}$ in diameter). Fused silica is used for all lenses. Good images require that there be at least two aspheric surfaces. In this particular design, both aspheres have been placed on element $\mathrm{C} 4$, although they can be split between $\mathrm{C} 2$ and $\mathrm{C} 4$ and achieve similar performance.

The design was optimized for good images in the four bandpasses listed in Table 1, allowing for a refocus for each bandpass.

The design uses a single glass type, for which many choices are possible. The choice of fused silica as the lens material is driven by three considerations. First, based on informal contacts with vendors, fused silica blanks are competitive with other types of glass when both cost and schedule are factored together. Second, fused silica is the only material that provides acceptable UV throughput, given the large lens thicknesses that we will use. Third, insofar as the dewar window is an active element in the corrector, fused silica meets our radiation requirement, whereas other materials (such as BK7) do not or would need to be validated first.

\section{POINT SPREAD FUNCTION}

Table 2 gives the breakdown of the expected contributions of the telescope, corrector, and detector to the point spread function (PSF). The requirement on the total contribution, 0.55 arcsec, is the estimated contribution from the existing telescope, Mosaic II instrument, and other effects such as "dome seein". This value was determined by comparing a histogram of site seeing with the actual measured PSF in a large number of images taken as part of the Super Macho project, which provides the best record of the delivered performance at the prime focus (Fig. 2). Our requirement for the PSF produced by the corrector itself is the same as that for the existing as-designed prime focus corrector.

Our requirements on PSF size are not as demanding as those of some other proposed correctors, so one might ask what the benefit would be of improving the performance. The answer is actually not much, for three reasons. First, the CCDs themselves contribute an equal amount to the PSF due to the effect of "lateral charge diffusion", 8 which is intrinsic to all CCDs but seldom discussed in PSF budgets. Lateral diffusion arises because a charge created by the absorption of a photon can migrate laterally on its way to being collected by a CCD pixel. The $1-\sigma$ width of the diffusion process can be several microns. For the LBL CCDs, we expect that it will be $7.5 \mu$ which corresponds to the FHWM listed in the table. This value is larger than the contribution from the corrector itself. Second, with our chosen pixel size of $15 \mu$, the smallest images that are properly sampled are about $0.6^{\prime \prime}$, which is the median site seeing. If the telescope and instrument were perfect, we would be undersampling the PSF half the time. Finally, in a survey, the success or failure of a survey is not determined by the best images possible but rather by the worst images that are still acceptable for the survey's scientific 


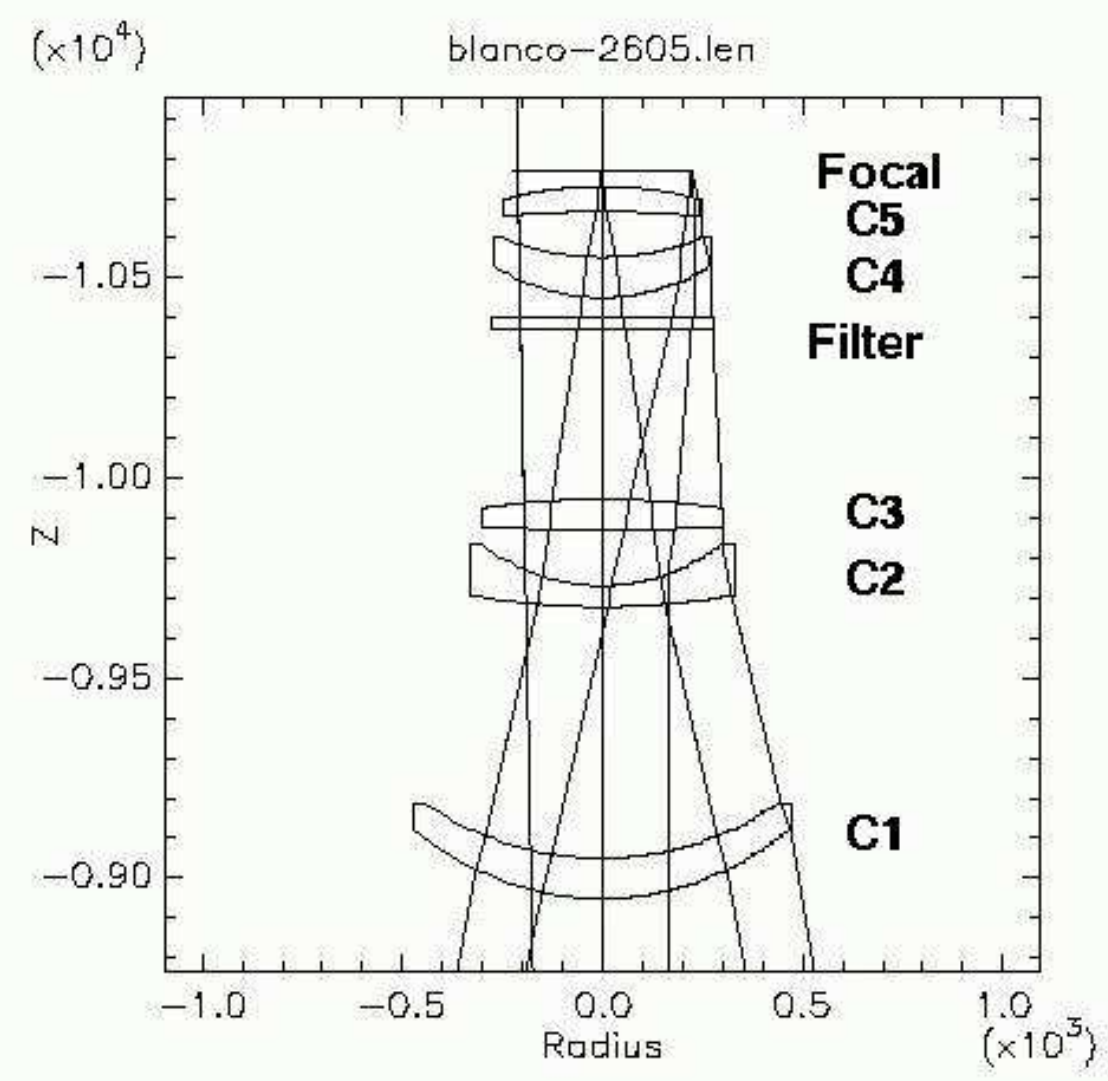

Figure 1. Layout of corrector design. Element C5 doubles as the dewar window.

goals. In our case, the worst acceptable images are 1.2" FHWM, with a goal that the median be 0.9" FHWM or smaller.

Although not directly relevant for the corrector design, an important component of the error budget is the contribution from the optical collimation of the telescope as a whole, plus any contributions from distortion of the primary mirror. The known contribution from the mirror figure and a contribution from a modest offset between the primary mirror optical axis and that of the corrector is included in the table, but any additional contributions are absorbed under "contingency". The current mirror support system is known to have some problems that allow the mirror to move laterally in its cell, but a program to improve the support system is in progress and should lead to an improved performance of the system in the future. ${ }^{9}$

\section{GHOSTING}

In an optical system such as this with a large number of lenses, particular attention needs to be paid to ghosting to prevent unanticipated side effects. Such attention is needed even if all surfaces are treated with high performance anti-reflection coatings. The silicon in CCDs, with a refractive index of 3.46, is highly reflective. The actual reflectivity is a sensitive function of wavelength and the details of the coating. The LBL CCDs are treated with a wide-band coating that gives reflectivities ranging from $5 \%$ to $20 \%$ over the wavelengths of the DES filters. We will assume an average reflectivity of $15 \%$ for the calculations that follow.

The lens coatings have not been finalized but will likely be a combination of Sol-Gel for most surfaces and a hard coating for the dewar window and any other exposed surfaces. For simplicity, we assume $1 \%$ reflectivity 


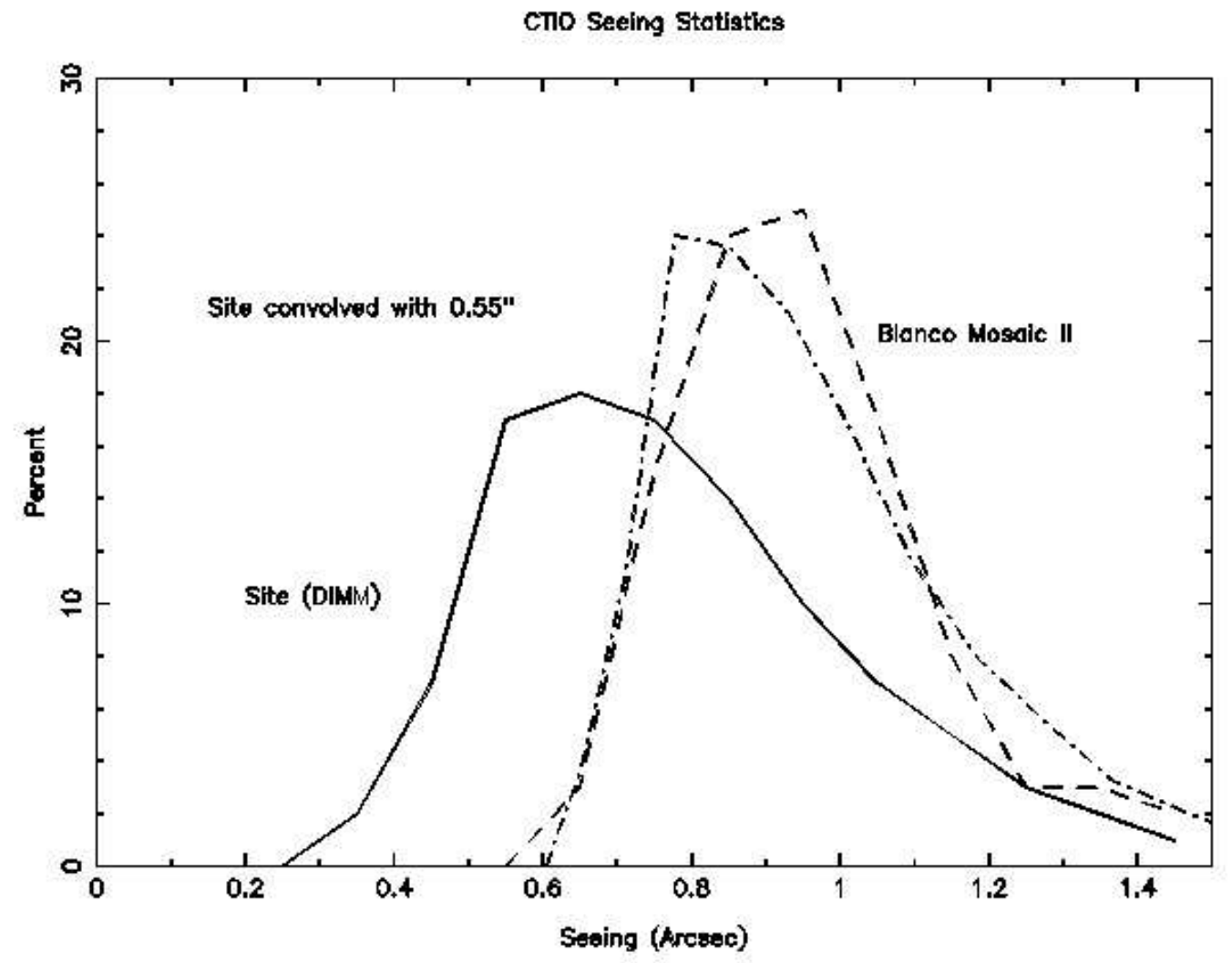

Figure 2. Point spread function FWHM for current CTIO $4 \mathrm{~m}$ Mosaic II camera, compared with site seeing

for the calculations that follow.

Ghost images are formed by a double-reflection between the CCDs in the focal plane and any one of the lens surfaces. Two types of ghost images are particularly pernicious: those from out-of-focus images around isolated bright stars, and those of in-focus images of the exit pupil illuminated by the night sky. Ghost images cause problems with both sky subtraction, which is important for faint objects, and flatfielding, which is important for photometric calibration of all objects if, as is normally done, one presumes that variations in the local sky signal directly measure the sensitivity of the detector and filter. We examine each of these ghosts in turn.

Out-of-focus ghost images of bright stars, such as produced by reflections from the dewar window, are ubiquitous, and one's choices for controlling them are limited to keeping them small and bright or allowing them to be large but diffuse. Our choice is to emphasize the latter. At high Galactic latitudes, there is an average of one 6th magnitude star per field. Our requirement is that such a star of apparent magnitude $m$ should produce a halo with a surface brightness of no more than $m+19 \mathrm{mag} \operatorname{arcsec}^{-2}$. The main impact of this requirement is to set a minimum distance of the dewar window from the focal plane.

The exit pupil ghost arises when the ghosting surfaces create an in-focus image of the exit pupil on the focal plane. In nearly all prime focus correctors, there is one surface that dominates; in our corrector, it is the leading surface of C4. One has two strategies for controlling such ghosts - either allow them to be precisely in focus and subtract them in the data processing software, or defocus them so they are innocuous on small angular scales where standard local sky subtraction can be done (Fig. 3). As an example of the former, the current corrector on the Mayall telescope creates an in-focus image of the exit pupil with an amplitude of order $1 \%$ of the total sky brightness. This image is removed using special routines in IRAF. As an example of the latter, the current corrector on the Blanco telescope creates a defocused image of the exit pupil; this image is sufficiently 
Table 2. PSF Budget

\begin{tabular}{|c|c|c|}
\hline Source & $\begin{array}{r}\text { FHWM (1-d) } \\
(\text { arcsec })\end{array}$ & \\
\hline Dome Seeing & & Absorb in "contingency" \\
\hline Telescope Guiding errors & 0.03 & Same as focus errors \\
\hline Wind Shake & 0 & "Assume "calm" night \\
\hline \multicolumn{3}{|l|}{ Corrector } \\
\hline Design & 0.26 & Average of $r, i$ \\
\hline Manufacturing tolerances & 0.1 & (Radii, index, thickness) \\
\hline Glass homogeneity & 0.065 & Striae, etc $2 . \mathrm{e}-6$ \\
\hline Assembly errors & 0.0571 & (decenter, tilt) \\
\hline Flexure & 0.035 & \\
\hline CCD Focal Plane flatness & 0.05 & Peak-peak 30 micron $z$ error \\
\hline Thermal performance & 0.0466 & Worst-case change of 30C (Steel) \\
\hline CCD Diffusion & 0.31 & $\begin{array}{l}\text { From LBNL tests - expected performance } \\
(7.5 \text { microns } 1-D)\end{array}$ \\
\hline Depth-of-focus in CCD & 0.03 & ( $i$ depth of focus only) \\
\hline Primary Mirror figure & 0.16 & CTIO mirror testing report \\
\hline Primary mirror support (static) & & Absorb in "contingency" \\
\hline Primary mirror support flexure & & Assume "active optics" are perfect \\
\hline Telescope Collimation (static) & 0.05 & 200 micron offset of primary mirror \\
\hline Telescope flexure/mirror translation & & Absorb in Contingency \\
\hline Focus & 0.03 & Scaled from $2.5 \mathrm{~m}$ performance \\
\hline TOTAL (Tel+Instrument) & 0.47 & \\
\hline Contingency & 0.29 & Primary mirror support, dome seeing, ... \\
\hline Requirement & 0.55 & \\
\hline
\end{tabular}

unnoticeable that it it ignored in data processing, although it undoubtedly affects the photometric calibration.

In our case, the option of creating an in-focus image of the pupil and subtracting it in software, using the Mayall experience as a guide, is unattractive for at least three reasons. First, the in-focus exit pupil image is aberrated and thus does not have as well-defined edges as the images from the Mayall corrector. Thus, it would be more difficult to construct a suitable mask for defining the pupil ghost in the first place. Second, the optical paths producing the ghost image are all downstream from the filter, so the ghost intensity is expected to be several percent. For the Mayall corrector, the light producing the ghost image makes a double-pass through the filter, so for standard Johnson filters with a peak transmission that is less than $100 \%$ and with gently tapered bandpass edges, the intensity of the ghost image is suppressed by a factor 2 or more relative to what it would be otherwise. Third, the fraction of focal plane area covered with CCDs in our corrector is higher than that for the Mayall Mosaic I camera, which will again result in a higher ghost image intensity.

Thus, we choose to adjust the design to defocus the ghost pupil image as much as possible. The condition on amount of defocus required is that the change in intensity of the exit pupil ghost produced by the ambient night sky across the long axis of a CCD be no more than $3 \%$ of the night sky intensity itself. The motivation for this requirement is that if one were to perform standard flatfielding of a single CCD and use a single gain for photometric calibration, the resulting error introduced would still be less than our requirement on calibration accuracy. Ideally, the calibration could be improved through the use of "star flats" that calibrate the large-scale sensitivity variations of the CCD detectors independently of the night sky or ghost signal. Our requirement on 

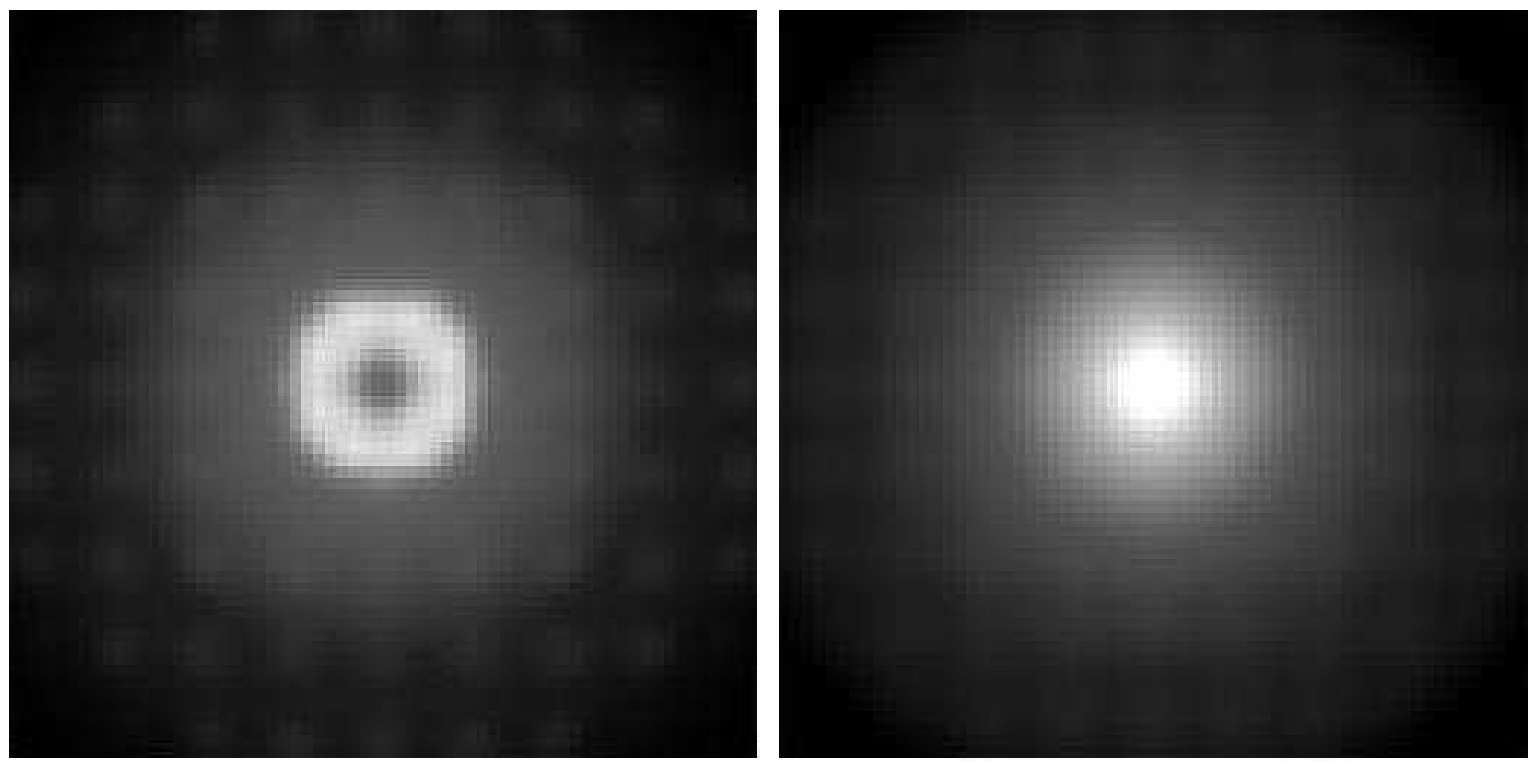

Figure 3. Image of exit pupil ghost. The figure on the left is an in-focus image of the exit pupil. The figure on the right is an image with the optics spaced so as to make the ghost image maximally out-of-focus while still producing acceptable image sizes at the corrector focal plane.

limiting the slope of the ghost image intensity makes such a calibration more tractable.

The minimization of exit pupil ghosting turned out to be somewhat tricky and required that C5 be moved away from the focal plane, resulting in a slight degradation of the overall PSF size. This is the one non-trivial tradeoff that we have made between PSF size and other factors in the design.

\section{WEAK LENSING REQUIREMENTS}

The goal of weak lensing science is to measure elliptical distortions in the images of galaxies induced by the gravitational influence of intervening matter. Because the intrinsic ellipticity of any galaxy image is unknown but significant, the weak lensing signal must be determined by averaging the apparent shapes of thousands of galaxies, on the assumption that intrisic shapes are uncorrelated. The apparent shapes must also be corrected for any contributions induced by the intrinsic point spread function (PSF) of the instrument, telescope, and atmosphere. Uncertainties in the intrinsic PSF shape are the largest source of systematic error in the weak lensing measurement. Thus, a qualititative requirement on the optics is that the PSF be stable and uniform across the focal plane.

The PSF is measured from the shapes of stars distributed across the focal plane. In any CCD exposure, the PSF is the convolution of contributions from static sources, such as the as-built corrector, and several dynamic (time-varying) sources, including the atmosphere, telescope tracking, gravity-induced telescope flexure, etc. While the weak lensing signal itself is an elliptical distortion of galaxy images, ellipticity is a poor parameter on which to place requirements because it is not preserved in the convolution process. Rather, we work directly with the second moments of the PSF, since these are additive (in quadrature) during convolution. By analogy with an ellipse, we define the "whisker" length $c$ to be

$$
c^{2}=\sqrt{\left(\left\langle x^{2}\right\rangle+\left\langle y^{2}\right\rangle\right)^{2}+4\langle x y\rangle^{2}},
$$

where $x$ and $y$ are coordinates measured relative to the PSF centroid and the angle brackets \langle\rangle denote expected values. If the PSF is elliptical with major and minor axes given by $a$ and $b$ respectively, then $c^{2}=a^{2}-b^{2}$, and $c$ 

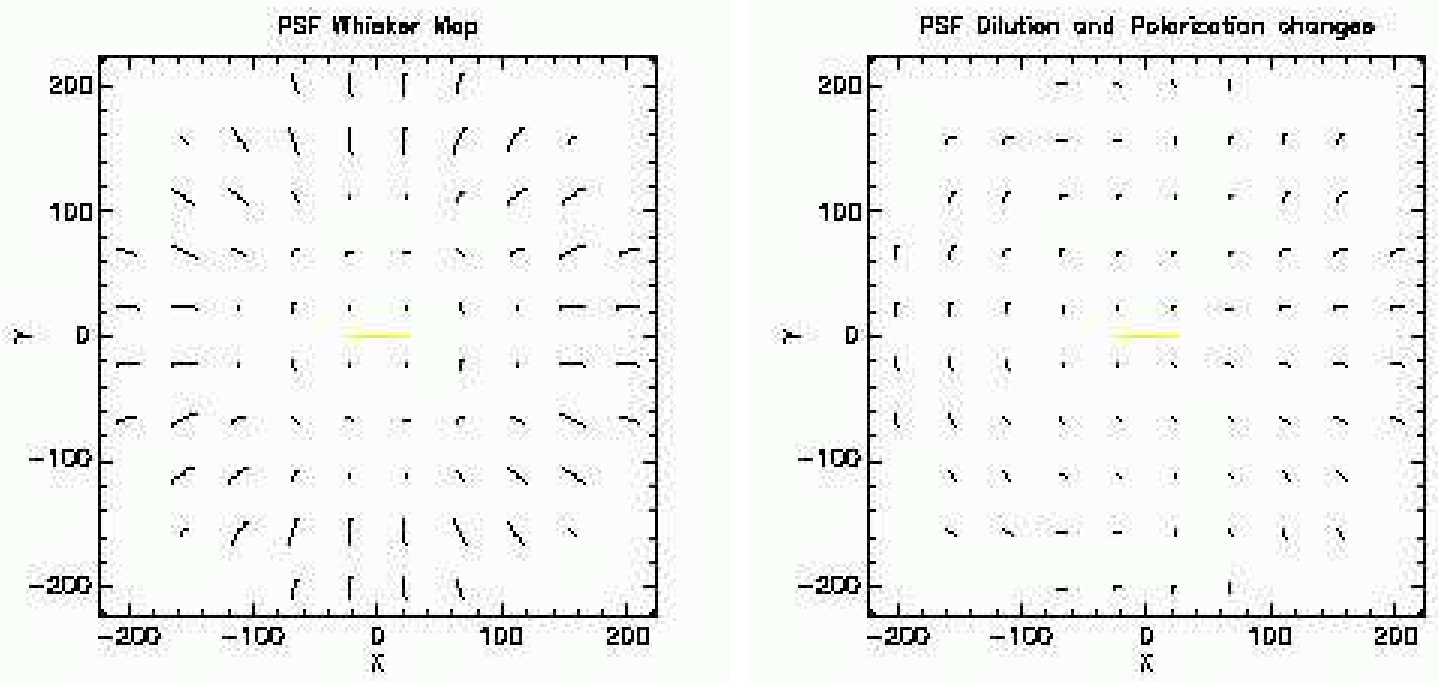

Figure 4. Whisker patterns produced by the corrector and mirror. The figure on the left shows the static pattern produced by the as-designed optics at a set of positions across the focal plane. The largest whisker has a length of $0.46^{\prime \prime}$. The figure on the right shows the change in whisker pattern induced by a decenter of the primary mirror of $100 \mu$. The largest whisker has a length of $0.16^{\prime \prime}$.

is the distance from the ellipse center to a focus. An elliptical PSF can thus be considered as resulting from the convolution of a spherical PSF with radius $b$ and a linear PSF with semi-axis $c .^{\ddagger}$

For the DES, we estimate that the $1-\sigma$ statistical error on measuring the average ellipticity (or shear) of all galaxies in a 2.2 degree diameter field is $0.1 \%$. If a typical galaxy has an intrinsic size (FWHM) of 1.2 arcsec (about the limit of resolution of the DES), the equivalent error in the whisker length (expressed as a FWHM) is 0.06 arcsec. We take this value to also be the maximum allowed systematic error in the PSF shape calibration; such a systematic error would further degrade the statistical error by of order an additional $15 \%$.

The accuracy with which the PSF can be calibrated depends quite a bit on the details of the calibration method. Normally, a PSF shape is computed for each exposure and each CCD in a mosaic camera independently. All medium bright stars in a field are used to compute a PSF shape, which is a two dimensional function of position on the CCD. In practice, the number of stars is seldom sufficient to fully map the PSF shape variations. Recently, techniques have been developed ${ }^{11}$ that allow one to combine data from multiple exposures from different telescope pointings to empirically determine the variation in PSF shape as a function of both space and time. The time variation is decomposed into a limited set of principal components that, in principle, should correspond to physically varying parameters of the telescope and optics that ultimately affect the PSF shape.

Figure 4 shows the whisker pattern for the as-designed focal plane and the change in the pattern due to a decenter of the primary mirror.

For the DES, the density of calibration stars will be of order one every 3 arcmin. Since the calibration plan is not yet known (and may not be decided until data are actually obtained), we have chosen not to place requirements on any static contributions to the PSF (although we do calculate them) and instead focus on dynamic contributions, with a goal being to place limits on those that can be controlled.

Table 3 gives the changes in whisker length of the PSF due to the corrector itself and a number of other factors that affect the PSF shape. The entry for "Static Pattern" gives the average whisker length itself. The

$¥$ We note that the dimensionless number $c / a$ is properly called the eccentricity, while the parameter $1-b / a$ is, following Hubble's convention, ${ }^{10}$ the ellipticity. The weak lensing community often abuses the terminology and uses the term ellipticity to refer to a different quantity; we refrain from such egregious usage. 
Table 3. Contributions to Whisker Length

\begin{tabular}{lcc}
\hline Source & Bandpass & $\begin{array}{c}\text { Average Whisker Differential } \\
\text { (arcsec) }\end{array}$ \\
& & \\
Static Pattern & $r, i, z$ & 0.32 \\
Spatial Pattern & $r, i, z$ & 0.09 \\
Differential Refraction & $r$ & 0.31 \\
(zenith angle 48 degrees) & $i$ & 0.18 \\
& $z$ & 0.10 \\
Defocus (10 microns) & ALL & 0.05 \\
Decollimation (100 microns) & ALL & 0.10 \\
& & \\
\hline
\end{tabular}

entry for "Spatial Pattern" gives the average varation in whisker shape in 3 arcmin steps across the focal plane. The dynamic factors that are considered are focus changes, decollimation, and differential refraction. It is seen that the peak-peak variation in whisker size is comparable to, or in some cases larger than, the maximum allowed systematic error. This means that the calibration procedure will need to measure and correct these dynamic and spatial variations in some fashion.

We have also computed whisker amplitudes and sensitivities for several variations of the corrector design and for correctors on other telescopes (including the current Mayall and Blanco correctors, LSST, VISTA, and Subaru). All have amplitudes and sensitivities roughly comparable to our corrector.

\section{ULTRAVIOLET PERFORMANCE}

While UV performance was not contrained in the optimization process, the corrector performance at these wavelengths across almost the entire focal plane is still respectable. The average FHWM is 0.5 arcsec. This performance, combined with the high throughput of fused silica, will allow observing programs in the UV to be conducted with the DES instrument. Although the CCDS are optimized for red performance, they still have moderate sensitivity ( $20 \%$ or more) well into the UV.

\section{CONCLUSIONS}

We have designed a corrector that meet the requirements of the DES, particularly with regard to field size and image quality. The corrector design has been kept simple in order to minimize cost while still providing good images at all wavelengths at zenith over the full area of the focal plane.

\section{REFERENCES}

1. B. Flaugher, "The Dark Energy Survey," Int. J. Mod. Phys. A. 20, pp. 3121-3123, 2005.

2. B. Flaugher, "The Dark Energy Survey Instrument Design," Proc. SPIE, this volume, 2006.

3. A. R. Smith, R. J. McDonald, D. C. Hurley, S. E. Holland, D. E. Groom, W. E. Brown, D. K. Gilmore, R. J. Stover, and M. Wei, "Radiation events in astronomical CCD images," in Sensors and Camera Systems for Scientific, Industrial, and Digital Photography Applications III, M. M. Blouke, J. Canosa, and N. Sampat, eds., Proc. SPIE 4669, pp. 172-183, 2002.

4. D. R. Blanco, G. Pentland, C. H. Smith, T. Dunham, and R. L. Millis, "Prime focus corrector for the Lowell 4-m telescope," in Specialized Optical Developments in Astronomy, E. Atad-Ettedgui and S. D'Odorico, eds., Proc. SPIE 4842, pp. 85-94, 2003. 
5. H. W. Epps and M. DiVittorio, "Preliminary optical design for a 4.0-m f/2.19 prime focus field corrector with a 2.0-degree field of view," in Specialized Optical Developments in Astronomy, E. Atad-Ettedgui and S. D'Odorico, eds., Proc. SPIE 4842, pp. 355-365, 2003.

6. P. Optics, "Blanco $4 \mathrm{~m}$ wide-field prime focus corrector (unpublished)," http://www.ctio.noao.edu /new/Telescopes/Blanco4m/NewInstruments/BlancoPrimeFocusCorrector-01.doc, 2003.

7. V. Terebizh, "A wide-field corrector at the prime focus of a Ritchey-Chre'tien telescope," Astron. Lett. 30, pp. 200-208, 2004.

8. A. Karcher, C. J. Bebek, W. F. Kolbe, D. Maurath, V. Prasad, M. Uslenghi, and M. Wagner, "Measurement of lateral charge diffusion in thick, fully depleted, back-illuminated CCDs," IEEE Trans. Nucl. Sci. 51, pp. 2231-2237, 2004.

9. T. Abbott, R. Catarutti, E. Mondaca, A. Montane, G. Schumacher, R. Tighe, A. Walker, D. Allspach, H. Cease, B. Flaugher, J. Peoples, A. Stefanik, J. Thaler, and V. Simatis, "Cerro Tololo Inter-american Observatory, Victor M. Blanco 4-m telescope and the Dark Energy Survey," Proc. SPIE, this volume, 2006.

10. E. Hubble, Realm of the Nebulae, Yale University Press, New Haven, 1936.

11. M. Jarvis and B. Jain, "Principal components analysis of PSF variation in weak lensing surveys," $A s$ troph/0412234, 2004. 